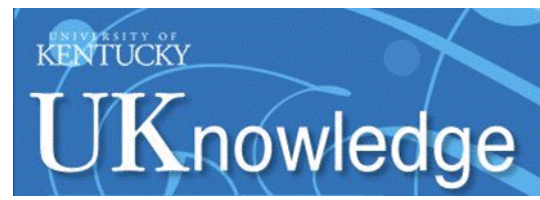

University of Kentucky

UKnowledge

$11-22-2017$

\title{
Load Model Verification, Validation and Calibration Framework by Statistical Analysis on Field Data
}

Xiangqing Jiao

University of Kentucky, xji227@uky.edu

Yuan Liao

University of Kentucky, yuan.liao@uky.edu

Thai Nguyen

LG\&E and Kentucky Utilities

Follow this and additional works at: https://uknowledge.uky.edu/ece_facpub

Part of the Electrical and Computer Engineering Commons, and the Statistics and Probability Commons

Right click to open a feedback form in a new tab to let us know how this document benefits you.

\section{Repository Citation}

Jiao, Xiangqing; Liao, Yuan; and Nguyen, Thai, "Load Model Verification, Validation and Calibration Framework by Statistical Analysis on Field Data" (2017). Electrical and Computer Engineering Faculty Publications. 18.

https://uknowledge.uky.edu/ece_facpub/18

This Article is brought to you for free and open access by the Electrical and Computer Engineering at UKnowledge. It has been accepted for inclusion in Electrical and Computer Engineering Faculty Publications by an authorized administrator of UKnowledge. For more information, please contact UKnowledge@lsv.uky.edu. 


\title{
Load Model Verification, Validation and Calibration Framework by Statistical Analysis on Field Data
}

\author{
Digital Object Identifier (DOI) \\ https://doi.org/10.1515/ijeeps-2017-0070 \\ Notes/Citation Information \\ Published in International Journal of Emerging Electric Power Systems, v. 18, issue 6, 20170070, p. 1-7. \\ (c) 2017 Walter de Gruyter GmbH, Berlin/Boston.
}

The copyright holder has granted the permission for posting the article here.

This article is available at UKnowledge: https://uknowledge.uky.edu/ece_facpub/18 
Xiangqing Jiao ${ }^{1}$ / Yuan Liao $^{1} /$ Thai Nguyen $^{2}$

\title{
Load Model Verification, Validation and
}

Calibration Framework by Statistical Analysis on Field Data

\author{
${ }^{1}$ University of Kentucky-ECE, Lexington, Kentucky, USA, E-mail: yliao@engr.uky.edu \\ ${ }^{2}$ Power Planning Division, LG\&E and Kentucky Utilities, Louisville, Kentucky, USA
}

\begin{abstract}
:
Accurate load models are critical for power system analysis and operation. A large amount of research work has been done on load modeling. Most of the existing research focuses on developing load models, while little has been done on developing formal load model verification and validation $(\mathrm{V} \& \mathrm{~V})$ methodologies or procedures. Most of the existing load model validation is based on qualitative rather than quantitative analysis. In addition, not all aspects of model $\mathrm{V} \& \mathrm{~V}$ problem have been addressed by the existing approaches. To complement the existing methods, this paper proposes a novel load model verification and validation framework that can systematically and more comprehensively examine load model's effectiveness and accuracy. Statistical analysis, instead of visual check, quantifies the load model's accuracy, and provides a confidence level of the developed load model for model users. The analysis results can also be used to calibrate load models. The proposed framework can be used as a guidance to systematically examine load models for utility engineers and researchers. The proposed method is demonstrated through analysis of field measurements collected from a utility system. Keywords: load modeling, statistical analysis, verification, validation, calibration
\end{abstract}

DOI: $10.1515 /$ ijeeps-2017-0070

Received: April 14, 2017; Accepted: November 10, 2017

\section{Introduction}

Computational simulation model of power system is the basis of power system study. Load modeling is critical for power system stability analysis and control [1-4]. Modeling loads, however, is a quite challenging task due to load's variability and uncertainty. A large amount of research work has been done on load modeling. Measurement-based and component-based methods have been developed to build load models [5-8]. These approaches mainly address two topics: load model structure determination and load model parameter estimation. However, a critical issue, load model verification and validation, has attracted much less effort. Little research has been done on load model validation, and formal validation methodologies or procedures have not been developed and defined [9].

The objective of load model verification and validation is to ensure that the developed model can faithfully replicate the actual power system behavior. If the mismatch between simulation results and actual system performance is significant, system operators may not know the true states of the system, and severe accidents may occur, such as power system instability, outages, and at worst, cascading and wide-area blackout. Besides, verification and validation is also meaningful for model calibration.

The way to implement model verification and validation is critical in determining validity and justification of simulation models. Most of the existing model validation methods visually check the mismatch between actual and simulated performance, and expresses validity in a qualitative way [10]. Simple graphical comparison may reflect outward disagreement between simulation results and field measurements, but it provides little quantitative representation of such mismatch. In addition, traditional methods make a simple binary pass/fail decision based on a rigorous criterion, which is not favored from an engineer's point of view. Model validity should be considered in terms of degree rather than a binary decision [11]. Moreover, some aspects of model verification and validation have not been covered by existing verification and validation approaches. In some literatures, the term "verification" and "validation" are considered as equivalent, while they have significantly different meanings.

In $[12,13]$, certain quantitative analysis is utilized in load model validation. In [14], quadratic errors between measured and simulated quantities are calculated, and the load models would be taken as valid if the 
quadratic errors are less than $5 \%$. The authors of [15] evaluate the developed model's performance by calculating relative error, and accepts the model if the relative error is less than $5 \%$. Reference [10] develops a load model validation method that quantifies the model's accuracy and uncertainty using accuracy index. He etal. proposes an approach to build composite load models via measurement-based approach [16]. The proposed method includes testing on developed load model's generalization capability, i.e. the model's ability to fit the newly collected measurements.

To complement existing methods, this paper proposes a framework for load model verification, validation, and calibration. This framework aims to serve as a systematic guidance for researchers and utility engineers, and covers some issues that have been inadequately studied. Statistical analysis indicating a certain level of confidence is used instead of binary pass/fail decision. The load model's generalization capability can be studied as well. Possible reasons leading to rejection of load model are discussed. The rest of the paper is organized as follows. Section 2 describes the framework and critical concepts involved, such as load model, verification, validation and calibration. Load model validation process based on statistical analysis is presented in Section 3. Model calibration is discussed in Section 4. A case study is presented in Section 5. Finally, the conclusion is drawn in Section 6.

\section{Framework description}

A load model is a set of mathematical equations that represent the relationship between voltage and/or frequency of a load and its real and reactive power consumption. Generally, voltage dependency is the main concern, while frequency dependency is usually ignored for simplicity [5]. The output of a load model is the real and reactive power consumed by the load with input being the voltage.

Load models can be built by using measurement-based approach or component-based approach. Measurement-based approach regards a load model as a black box model, and determines load type and model parameters based on field measurements. Component-based method identifies individual load models, and aggregates them into a single load.

Accurate load models are critical for power system analysis, operation and control. In the past, a lot of load models approximate loads as static models which neglect loads' dynamic properties [6]. Given that the dynamics characteristics of loads have a major impact on system stability, static load models are increasingly considered as inadequate for dynamic studies. The existing load models need to be regularly verified and validated.

The definitions of verification and validation were originally formalized by the Defense Modeling and Simulation Office of the United States Department of Defense [17]. To make such concept clearer from the perspective of physics and engineering communities, the Computational Fluid Dynamics Committee on Standards of the American Institute of Aeronautics and Astronautics (AIAA) stated these concepts as follows [18]:

- Verification is the process of determining that a model implementation accurately represents the developer's conceptual description of the model and the solution to the model.

- Validation is the process of determining the degree to which a model is an accurate representation of the real world from the perspective of the intended uses of the model.

In the context of load model, verification process should verify that the load model faithfully represents the assumption of the load model. Therefore, the codes and parameters of load models should be checked to ensure that the load type and parameters are set as assumed. For example, if a load model is assumed to be a ZIP load, the model's mathematical expressions of the constant impedance, constant current, and constant power load should be checked. Not only the load composition, but also the portions and parameters for each type should be true to the assumption. If the model is considered unacceptable, the reason why the model is rejected should be identified, and corresponding modification should be made before proceeding to model validation.

Load model validation process validates that the assumption of model structure and model parameters are representative of the actual system load. The simulated output of load models should be compared with the measured output of actual loads. Based on statistical analysis of such comparison, a conclusion on whether to accept or reject the load models can be drawn. Note that since both the load type and load scale can change over time, a universal load model adapting to all conditions does not exist. Even if the model is valid for a certain system condition, it may not be able to represent actual loads at a later time. Therefore, the model's ability to reflect system load's behavior in unseen conditions, which is known as generalization capability, needs to be examined as well. If multiple sets of measurements or historical data are available, model's generalization capability could be validated based on the data recorded under different system conditions. If the load is strongly dependent on a certain factor, such as season, weather, etc., different models can be built for different scenarios. 
For example, electric heating loads vary seasonally, so different load models can be built for different seasons. The model or models for a load should be valid for most scenarios, but it is acceptable if these models do not fit some situations.

Based on the verification and validation results, model calibration can be made. Calibration is defined by AIAA as a process of adjusting parameters in the computational model for the purpose of improving the agreement between model results and measurement or experiment data [18]. Reasons to reject a load model will be discussed in Section 3, and corresponding changes can be made.

Figure 1 shows the proposed verification, validation and calibration framework. After obtaining the latest load model, verification process is implemented. If the model is verified to be true to the conceptual model, validation study based on field measurements is consequently implemented. If the model does not pass either verification or validation test, the original model should be calibrated. Both $\mathrm{V} \& \mathrm{~V}$ analysis results and field measurements can be used to aid model calibration.

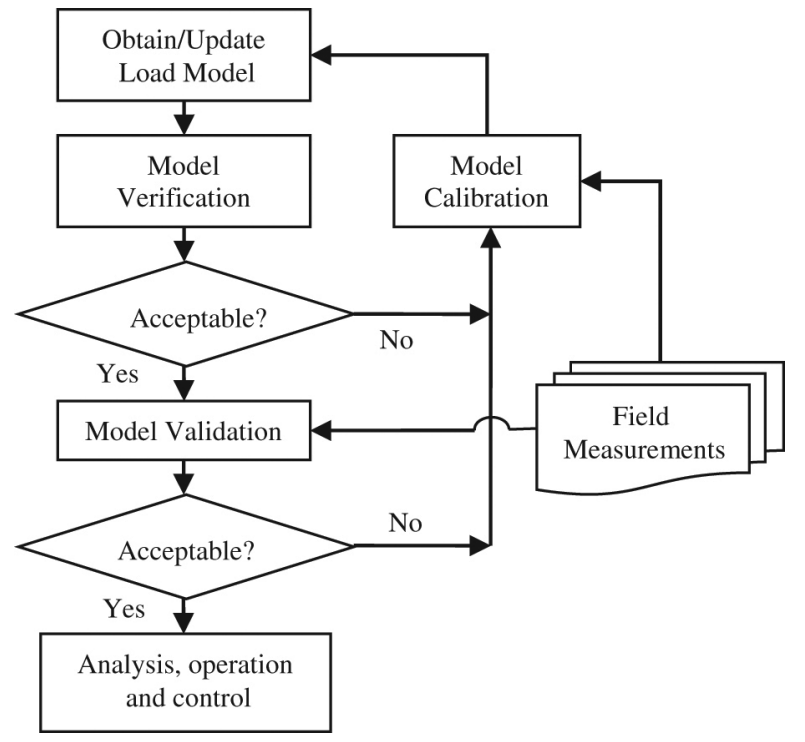

Figure 1: Load model verification, validation and calibration framework.

The key in model validation is comparison of simulated results and measurements. The following section will present validation work based on statistical analysis.

\section{Model validation based on statistical analysis}

Load model validation ensures that the load model can represent the real world load. Validation is critical for many studies, particularly voltage stability.

To examine the model's level of accuracy, field measurements should be obtained. Measurements from load aggregation point would be ideal for load model validation. In this way, the effects of other components' models on system states can be excluded. If such measurements are not available, measurements from other buses are still proficient under the assumption that the other components in the power system, in addition to the studied load, are modeled correctly.

The proposed validation method is based on online disturbance monitoring or ambient monitoring. Disturbances such as short-circuit faults and loss of generation usually cause large voltage variations, and can be used for dynamic model validation. Such disturbance can be either natural disturbance or man-made testing event. The use of natural disturbance data is advantageous from a technical and economical point of view. In addition to disturbance data, normal operation data could also be used in load modelling. First, the steady state data can be used to validate model's steady-state responses. Second, daily normal operation involves voltage variations as well, and these voltage variations can be used to validate model's dynamic characteristics. For example, voltage variations at the load aggregation point may be caused by switching on/off capacitors that are used to compensate reactive power, tap changer operations, etc. These voltage variations during normal operation can also be used to validate load models.

Load model validity is confirmed if matching degree between the simulated response and the measured response is high enough. If there are significant differences between the load's actual behavior and the model's prediction, the model is considered to be invalid, and calibration is required. The degree of agreement is obtained based on classical statistical methods. The eventual goal of model validation is to ensure that the model 
can reasonably predict load's output. Therefore, it would be desirable to validate the model performance over a wide spectrum of events, if measurements under different scenarios are available.

The validation process can be accomplished in the following steps:

- Step 1: Data collection: install measuring devices in the system (ideally at load aggregation point), and obtain measurements of desired quantities.

- Step 2: Event identification and replication: identify suitable events and develop power flow case to represent system conditions immediately prior to the event.

- Step 3: Model execution and result comparison: run dynamics simulation of the selected disturbance, and compare simulated response with actual response based on statistical method.

- Step 4: Generalization capability test: if multiple sets of measurements are available, repeat validation procedure with available recordings under a range of different conditions.

Hypothesis testing based method and confidence interval based method are classical techniques to quantitatively validate model performance. Hypothesis testing and confidence interval estimation are related methods, and both can be used to acquire a quantitative representation of model validity. Confidence interval provides a numerical answer to how close the simulated output is to the actual output [19]. Therefore, statistical analysis based on confidence interval is adopted in the framework. A confidence interval is an interval of values computed from sample data that is likely to include the unknown value of a population parameter [19]. In the context of load model validation, the sample data is the mismatch between simulated response and the measured response to the recorded events, and the population parameter refers to the mismatch between the simulated response and the actual response to all conditions. For a confidence interval, the confidence level is the probability that the computed interval will include the population parameter. Confidence level is generally expressed as a percentage, and the most common confidence level used by researchers is $95 \%$. Significance test accepts or rejects a model based on the $95 \%$ confidence interval. Such statistical validation result will help model users to decide model's validity quantitatively.

Assume that in a recorded event there are $N$ time instants. At each time instant, an actual output value and a simulated output value is recorded. Absolute error is adopted to measure the difference between the estimated value (simulated response) and what is estimated (actual response). Absolute error can be computed based on the following equation.

$$
A E_{i}=\left|\frac{X_{i m}(\theta)-X_{i s}(\theta)}{X_{i s}(\theta)}\right|, i=1, \ldots, N
$$

where $|$.$| represents absolute value of its argument, X_{i m}$ is the measured output at the $i$ th time point, $X_{i s}$ is the simulated output at the $i$ th time point, $N$ is the total number of time points, and $\theta$ is the set of parameters of load model. The mean absolute error can then be obtained as

$$
M A E=\frac{1}{N} \sum_{i=1}^{N}\left|\frac{X_{i m}(\theta)-X_{i s}(\theta)}{X_{i s}(\theta)}\right|
$$

The sample standard deviation $\sigma$ can be determined as

$$
\sigma=\sqrt{\frac{1}{N} \sum_{i=1}^{N}\left(A E_{i}-M A E\right)^{2}}
$$

The uncertainty level of the model is to be expressed by confidence interval within which the true values of the absolute error is expected to lie with a specified level of confidence. The confidence interval can be obtained as

$$
\text { Confidence Interval }=\text { MSE } \pm \text { Margin of Error }
$$

where the margin of error (MOE) can be determined by the following equation

$$
\text { MOE }=\text { Critical Value } \times \sigma
$$


In eq. (5), the critical value determines the amount of confidence we have in the result. For a $95 \%$ confidence interval, the critical value is 1.96 , and it can be rounded off to 2 . Finally, the confidence interval is calculated based on eq. (4).

If the computational load model ideally represents the characteristics of actual load, the simulated outputs will be the same as measured outputs, i.e. the absolute errors between the simulated and measured output are zero. Confidence interval gives a plausible range of such difference. All values in the confidence interval are plausible values, whereas values outside the interval are rejected as plausible values for the difference. Therefore, if the confidence interval does not include zero, it can be concluded that the measured outputs are significantly different from the simulated outputs. The hypothesis that the model is valid should be rejected.

If multiple sets of measurements are available, the model's generalization capability can be examined. Generalization capability of a model is the model's ability to reflect the load's behavior under unseen conditions [16]. By repeating validation process based on measurements taken under various scenarios, the model's generalization capability can be estimated.

\section{Model calibration}

Based on the verification and validation results, model calibration is implemented. There are a few reasons that could lead to rejection of a load model:

- Rejection reason I: model does not pass verification test. Errors are identified in model codes.

- Rejection reason II: assumed load model structure is not suitable.

- Rejection reason III: assumed load model parameters are not accurate, while the load model structure is suitable.

- Rejection reason IV: wrong measurements are used, e.g., phase A voltage should have been used, but phase $B$ voltage is actually used.

- Rejection reason V: measurement errors are significantly large, and thus affect the validation results.

- Rejection reason VI: the model of other components in the system is wrong, which leads to erroneous simulated model outputs.

- Rejection reason VII: the model is not suitable for the specified scenario.

Reason I involves with verification process. It should be examined before validation process implementation, and mistakes in model codes should be corrected. Rejection type II and III are straightforward: inaccurate model parameters and inappropriate model structure would almost certainly result in inaccurate responses. To calibrate the mode, a suitable model structure that is representative of the actual load should be selected. Non-linear least square based optimization algorithms can be used for parameters estimation. Type IV and V rejection are involved with measurements instead of load model. Type VI rejection is involved with external component's model instead of load model. Types IV-VI should be checked primarily in validation process to exclude the impacts of external factors. Type VII rejection addresses the model's generalization capability. It is acceptable if the model does not work for some particular situations. If necessary, dedicated model representing a particular situation can be built and used whenever such situation occurs. More advanced model calibration techniques will be developed in the future research.

\section{Case study}

This section presents an example of applying the proposed framework for load model verification, validation and calibration. The studied system is modeled in PSS/E, and its load model is to be verified and validated.

The event recorded and used is a single line to ground fault occurred on a bus. The fault lasts for eight cycles before being cleared. The positive sequence voltage magnitude at a generator bus is recorded and used for validation. The load model is type 'CMLDARU1 which is based on the composite load model proposed by the Western Electricity Coordinating Council.

Following the framework proposed in Section 2, model verification is implemented primarily. To verify that the existing model faithfully represent the assumptions, the parameters of the existing model are checked, such 
as the percentage of constant torque motors, load MVA bases, load tap changer transformer's tap adjustments, feeder impedance, and protection settings, etc. After checking model type implemented and the parameters used, it is confirmed that the existing model matches the users' conceptual description.

After model verification, model validity needs to be assessed. Figure 2 depicts the recorded voltage magnitudes and simulated voltage magnitudes based on the existing load model. It can be observed that the voltage sag in simulation is obviously greater that the measured value, and the simulated steady-state voltage after fault is also significantly different. Based on the observed difference, parameters involving motor proportion, protection setting, motor parameters, etc. are tuned. The details of model calibration are not presented in this paper, because it is out of this paper's scope. Figure 3 shows the comparison between the recorded voltage and the simulated voltage based on the calibrated voltage. The simulated voltage sag and the post-fault steady-state voltage coincide with the measurements. There are still some discrepancies between the two sets of data. The statistics of the default model and the calibrated model are listed in Table 1. The MAE of the default model is significantly greater than the calibrated model. The confidence interval of the default model does not include zero, while the confidence interval of the calibrated model does. Therefore, we can conclude that the default model is significantly different from the actual load, and we are $95 \%$ confident that the calibrated model represents the actual load's performance. It is noted that when multiple records of events are available, optimization based methods such as genetic algorithm, least squares method, etc., may be used to estimate or tune the values of the load parameters. If these records represent typical scenarios of concern, the optimizaton process may enhance the generalization ability of the load model.



Figure 2: Recorded voltage and simulated voltage based on the existing load model.

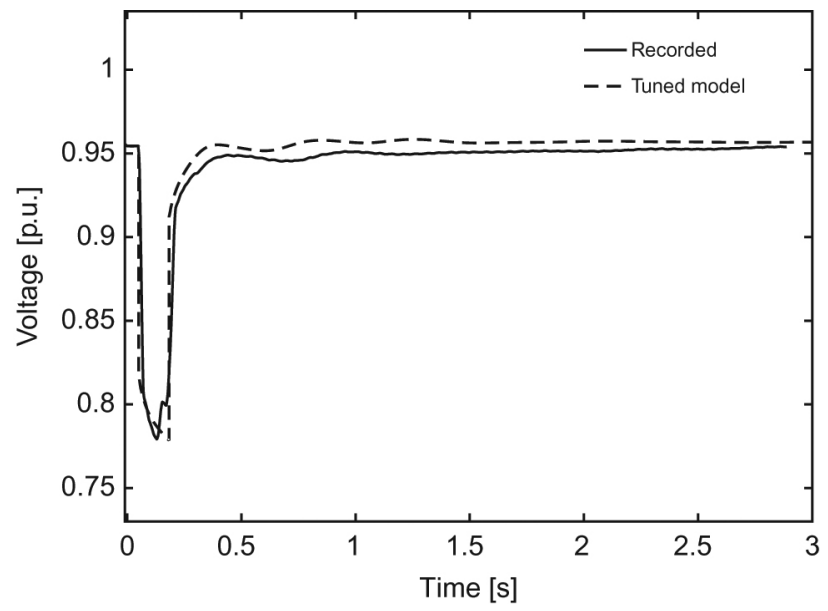

Figure 3: Recorded voltage and simulated voltage based on the calibrated load model.

Table 1: Statistics of dedault model and calibrated model.

\begin{tabular}{lll}
\hline Statistics & Default Model & Calibrated Model \\
\hline MAE & 0.0273 & 0.0071 \\
$\sigma$ & 0.0134 & 0.0118 \\
Critical Value & 1.96 & 1.96
\end{tabular}




\section{Conclusion}

A verification, validation, and calibration framework for load model is proposed in this paper. This framework fills the gap of formal load model V\&V methodology, and can be used as a guideline to help utility engineers to examine existing load models. In the framework, the model's validity is expressed in a quantitative way, which complements traditional graphical inspection method. A confidence level, rather than a binary pass / fail decision, is provided by the framework to quantitatively support the model user's decision. The verification and validation results can also be used to calibrate the model. Likely reasons that may result in model rejection are discussed. Evaluation study based on field measurements are presented to demonstrate application of the proposed methods.

\section{References}

[1] IEEE Task Force on Load Representation for Dynamic Performance. Load representation for dynamic performance analysis. IEEE Trans Power Syst. 1993;8(2):472-82.

[2] IEEE Task Force on Load Representation for Dynamic Performance. Bibliography on load models for power flow and dynamic performance simulation. IEEE Trans Power Syst. 1995;10(1):523-38.

[3] IEEE Task Force on Load Representation for Dynamic Performance. Standard load models for power flow and dynamic performance simulation. IEEE Trans Power Syst. 1995;10(3):1302-13.

[4] Cebreselassie A, Chow ]H. Investigation of the effects of load models and generator voltage regulators on voltage stability. Int ] Elect Power Energy Syst. 1994;16(2):83-89.

[5] Kundur P. Power system stability and control. New York: McCraw-Hill, 1993.

[6] Zhang P, “Measurement-based load modeling,” EPRI, Palo Alto, CA, Rep. 1014402, 2006.

[7] Vaahedi E, Fl-Kady MA, Libaque-Esaine ]A, Carvalho VF. Load models for large-scale stability studies from end-user concumption. IEEE Trans Power Syst. 1987;2(4):864-70.

[8] Hiskens IA, Milanovic JV. Load modeling in studies of power system damping. IEEE Trans Power Syst. 1995;10(4):1781-88.

[9] Pourbeik P. Approaches to validation of power system models for system planning studies. IEEE PES Ceneral Meeting, Minneapolis, MN, 2010: 1-10.

[10] Liang Y, Tam KS, Broadwater R. Load calibration and model validation methodologies for power distribution systems. IEEE Trans Power Syst. 2010;25(3):1393-401.

[11] Trucano TC, Swiler LP, Igusa T, Oberkampf WL, Pilch M. Calibration, validation, and sensitivity analysis: what's what. Reliability Eng Syst Safety. 2006;91:1331-57.

[12] Wang H, Ma ], Zheng X. Comparison and verification of load model based on PSAP. In: 2010 5th International Conference on Critical Infrastructure, Beijing, 2010: 1-5.

[13] Ye ], Ling D, He R, Pang S. Load modeling and validity verification based on the cluster analysis. In: 2012 International Conference on Electrical and Computer Engineering, Dhaka, 2012: 82-88.

[14] Aguero ]L, Barbieri MB, Beroqui MC. Voltage depending load models. Validation by voltage step tests. IEEE Power Engineering Society Ceneral Meeting, Montreal, Que., 2006: 1-6.

[15] Choi BK, Chiang HD, Li Y, Li H, Chen YT, Huang DH, et al. Measurement-based dynamic load models: derivation, comparison, and validation. IEEE Trans Power Syst. 2006;21(3):1276-83.

[16] He R, Ma ], Hill D]. Composite load modeling via measurement approach. IEEE Trans Power Syst. 2006;21(2):663-72.

[17] United State Department of Defense. DoD Directive No. 5000.59: modeling and simulation (M\&S) management, defense modeling and simulation office. Office of the Director and Defense Research and Engineering, 1994.

[18] American Institue of Aeronautics and Astronautics. Guide for the verification and validation of computational fluid dynamics simulations. AIAA, Reston, VA, AIAA-G-077-1998, 1998.

[19] Utts]M, HeckardRF. Mind on statistics, 4th ed. Boston: Brooks/Cole, 2011. 